\title{
The flavonoid $p$-hydroxycinnamic acid exhibits anticancer effects in human pancreatic cancer MIA PaCa-2 cells in vitro: Comparison with gemcitabine
}

\author{
MASAYOSHI YAMAGUCHI ${ }^{1}$, TOMIYASU MURATA ${ }^{2}$, BASSEL F. EL-RAYES ${ }^{1}$ and MAMORU SHOJI ${ }^{1}$ \\ ${ }^{1}$ Department of Hematology and Medical Oncology, Emory University School of Medicine, \\ Winship Cancer Institute, Atlanta, GA 30322, USA; ${ }^{2}$ Laboratory of Analytical Neurobiology, \\ Faculty of Pharmacy, Meijo University, Tempaku, Nagoya 468-8503, Japan
}

Received June 23, 2015; Accepted August 7, 2015

DOI: $10.3892 /$ or.2015.4282

\begin{abstract}
Pancreatic cancer is a highly aggressive malignancy with a notoriously dismal prognosis. A major contributor to this poor clinical outcome is pancreatic cancer's prominent chemoresistance. The present study was undertaken to determine whether the flavonoid $p$-hydroxycinnamic acid (HCA), which is a botanical factor, possesses anticancer effects on cloned human pancreatic cancer MIA PaCa-2 cells that possess resistance to radiation therapy in vitro. Proliferation of MIA PaCa-2 cells was suppressed after culture with HCA (10-1,000 nM). Such an effect was also noted in human pancreatic cancer $\mathrm{Pt} 45 \mathrm{P} 1$ cells. In the MIA PaCa- 2 cells, HCA induced $\mathrm{G} 1$ and $\mathrm{G} 2 / \mathrm{M}$ phase cell cycle arrest in the cells. The suppressive effects of HCA on proliferation were suggested to be mediated through the inhibition of various signaling pathways related to nuclear factor $-\kappa \mathrm{B}(\mathrm{NF}-\kappa \mathrm{B})$, extracellular signal-regulated kinase (ERK), protein kinase $C$, phosphatidylinositol 3-kinase (PI3K) or nuclear transcription activity. Moreover, HCA was found to stimulate cell death in the MIA $\mathrm{PaCa}-2$ and Pt45P1 cells in vitro. The anticancer effects of HCA on MIA PaCa-2 cells were exhibited at a lower concentration than gemcitabine, a potent cancer drug. The flavonoid HCA may be a useful tool in the therapy of human pancreatic cancer in vivo.
\end{abstract}

\section{Introduction}

The normal pancreas is made up of two classes of cells: endocrine (hormone-secreting) and exocrine (digestive

Correspondence to: Dr Masayoshi Yamaguchi, Department of Hematology and Medical Oncology, Emory University School of Medicine, Winship Cancer Institute, 1365-C Clifton Road NE, Atlanta, GA 30322, USA

E-mail: yamamasa1155@yahoo.co.jp

Key words: $p$-hydroxycinnamic acid, pancreatic cancer, MIA PaCa-2 cells, Pt45P1 cells, cell proliferation, apoptotic cell death enzyme-producing). Depending on the cell of origin, pancreatic cancers can be classified as endocrine or exocrine tumors. Roughly $90 \%$ of all pancreatic cancers are pancreatic ductal adenocarcinomas, an exocrine pancreatic tumor that resembles the cells lining the pancreatic duct (1). Pancreatic cancer is a highly aggressive malignancy with a notoriously dismal prognosis. Pancreatic cancer is the 12th most common cancer. Despite not being one of the most prevalent cancers, it is by far one of the deadliest, with a 5-year survival of less than $7 \%(1,2)$. It ranks fourth in terms of cancer mortality and accounts for less than $7 \%$ of all cancer-related death $(1,2)$. A major contributor to this poor clinical outcome is pancreatic cancer's prominent chemoresistance (3).

The flavonoid $p$-hydroxycinnamic acid (HCA), which is an intermediate-metabolic substance in plants and fruits, is synthesized from tyrosine $(4,5)$. HCA has been found to exhibit anabolic effects on bone metabolism in vitro and preventive effects on bone loss in osteoporosis model animals including postmenopause and type 1 diabetes in vivo (6-8). Among the botanical factor cinnamic acid-related compounds (cinnamic acid, HCA, ferulic acid, caffeic acid and 3,4-dimethoxycinnamic acid), HCA has been shown to possess a specific anabolic effect on bone metabolism in vitro (6). HCA has been shown to possess suppressive effects on osteoclastogenesis by antagonizing the receptor activator of nuclear factor- $\kappa \mathrm{B}$ ligand (RANKL)-induced NF- $\kappa \mathrm{B}$ activation and potent stimulatory effects on osteoblastogenesis and mineralization through inhibition of tumor necrosis factor (TNF)- $\alpha$-enhanced NF- $\kappa \mathrm{B}$ signaling in vitro (9-11). HCA was also found to suppress adipogenesis in bone marrow cells by inhibiting MEK/extracellular signal-regulated kinase (ERK) signaling in vitro (12). Thus, HCA has been demonstrated to exhibit suppressive effects on multi-signaling pathways in various types of cells to restore metabolic disorder.

It is well-known that many signaling pathways are attenuated in cancer cells. The effects of HCA on cancer cells have not been investigated. We hypothesized that HCA may exhibit anticancer effects on human pancreatic cancer MIA PaCa-2 cells in vitro, which possess resistance to drugs and radiation in pancreatic cancer therapy. This study was undertaken to determine whether HCA possesses suppressive effects on 
pancreatic cancer cells in vitro. HCA was found to suppress the proliferation and to stimulate apoptotic cell death of human pancreatic cancer MIA $\mathrm{PaCa}-2$ and $\mathrm{Pt} 45 \mathrm{P} 1$ cells in vitro. In this study, botanical factor HCA was demonstrated to possess anticancer cell effects.

\section{Materials and methods}

Materials. Dulbecco's modified Eagle's medium (DMEM) with $4.5 \mathrm{~g} / 1$ glucose, L-glutamine and sodium pyruvate and antibiotics (penicillin and streptomycin) were purchased from Corning Cellgro (Mediatech, Inc., Manassas, VA, USA). $\alpha$-minimum essential medium ( $\alpha$-MEM) was purchased from Sigma-Aldrich (St. Louis, MO, USA). Fetal bovine serum (FBS) was from HyClone Corp. (Logan, UT, USA). HCA (100\% pure) was obtained from Wako Pure Chemical Co., Ltd. (Osaka, Japan). TNF- $\alpha$ was from R\&D Systems (Minneapolis, MN, USA). PD98059, staurosporine, Bay K 8644, wortmannin or 5,6-dichloro-1- $\beta$-D-ribofuranosylbenzimidazole (DRB), sodium butyrate, roscovitine, sulforaphane, caspase-3 inhibitor and all other reagents were purchased from Sigma-Aldrich unless otherwise specified. Gemcitabine was obtained from Hospira, Inc. (Lake Forest, IL, USA). Gemcitabine and caspase-3 inhibitor were diluted in phosphate-buffered saline (PBS) and other reagents were dissolved in $100 \%$ ethanol for use in the experiments.

Pancreatic cancer cells. We used human pancreatic cancer MIA Paca-2, Pt45P1 (highly expressing tissue factors; high $\mathrm{TF}$ ) and Pt45P1 (highly expressing alternative spliced variant TF; asTF) cells $(13,14)$. These human pancreatic cancer cell lines were obtained from the American Type Culture Collection (ATCC; Rockville, MD, USA). MIA PaCa-2 cells are commonly used as a model for human pancreatic cancer cells. This cancer cell line possesses resistance for the therapy with drugs and radiation. This cell line was a useful tool to determine whether or not HCA exhibits anticancer effects. Therefore, we used the cell line. Moreover, we used Pt45P1 cells that highly express tissue factors.

Cell proliferation. Pancreatic cancer cells $\left(1 \times 10^{5} / \mathrm{ml}\right.$ per well) were cultured in a 24-well plate with DMEM containing $10 \% \mathrm{FBS}$ and $1 \% \mathrm{P} / \mathrm{S}$ in the absence or presence of HCA $(10,100,250,500$ or $1,000 \mathrm{nM})$ for $1,3,7$ or 14 days in a water-saturated atmosphere containing $5 \% \mathrm{CO}_{2}$ and $95 \%$ air at $37^{\circ} \mathrm{C}(15,16)$. In separate experiments, pancreatic cancer cells $\left(1 \times 10^{5} / \mathrm{ml}\right.$ per well $)$ were cultured in DMEM containing $10 \% \mathrm{FBS}$ and $1 \% \mathrm{P} / \mathrm{S}$ in the presence of sodium butyrate (10 and $100 \mu \mathrm{M})$, roscovitine (10 and $100 \mathrm{nM})$, sulforaphane (1 and $10 \mathrm{nM}), \mathrm{TNF}-\alpha(1 \mathrm{ng} / \mathrm{ml})$, Bay K $8644(1 \mu \mathrm{M})$, PD98059 $(1 \mu \mathrm{M})$, staurosporin $(0.1 \mu \mathrm{M})$, wortmannin $(1 \mu \mathrm{M})$, DRB $(1 \mu \mathrm{M})$, or gemcitabine $(100 \mathrm{nM})$ for 3-7 days. After culture, the cells were detached from each culture dish and counted.

Cell death. Pancreatic cancer cells $\left(1 \times 10^{5} / \mathrm{ml}\right.$ per well) were cultured using a 24-well plate in DMEM containing 10\% FBS and $1 \% \mathrm{P} / \mathrm{S}$ in the absence of HCA for 7 days. When reaching confluency, the cells were additionally cultured in the presence of HCA $(10,100,250,500$ or $1,000 \mathrm{nM})$ with or without gemcitabine $(100 \mathrm{nM})$ or caspase-3 inhibitor $(5 \mu \mathrm{M})$ for 3 or 7 days (17). After culture, the cells were detached from each culture dish.

Cell counting. After trypsinization of each culture dish using $0.2 \%$ trpysin plus $0.02 \%$ EDTA in $\mathrm{Ca}^{2+} / \mathrm{Mg}^{2+}$-free PBS for $2 \mathrm{~min}$ at $37^{\circ} \mathrm{C}$, the detached cells from the dish were collected after centrifugation (15-18). The cells were resuspended in PBS solution and stained with eosin. Cell numbers were counted under a microscope using a hemocytometer plate. For each dish, we took the average of two countings. Cell number was expressed as the number per well of the plate.

Statistical analysis. Statistical significance was determined using GraphPad InStat version 3 for Windows XP (GraphPad Software Inc., La Jolla, CA, USA). Multiple comparisons were performed by one-way analysis of variance (ANOVA) with Tukey-Kramer multiple comparisons post test for parametric data as indicated. $\mathrm{P}<0.05$ was considered to indicate a statistically significant difference.

\section{Results}

HCA suppresses the proliferation of pancreatic cancer cells. To determine whether HCA possesses suppressive effects on the proliferation of cloned human pancreatic cancer MIA $\mathrm{PaCa}-2$ cells in vitro, the cells were cultured in the presence of HCA (10-1,000 nM) for 1, 3, 7 and 14 days (Fig. 1). Cell numbers were elevated with increasing culture time. Addition of HCA suppressed the increase in cell number. Thus, HCA was found to exhibit suppressive effects on the proliferation of MIA PaCa- 2 cells. Moreover, we determined whether HCA possesses anticancer effects on the proliferation in other human pancreatic cancer cells. We used Pt45P1 (highly expressing tissue factors; high TF) and Pt45P1 cells (highly expressing alternative spliced variant tissue factor; asTF). The suppressive effects of HCA on the proliferation were also noted in the Pt45P1 (Fig. 2A) and Pt45P1 cells (Fig. 2B) in vitro, when cells were culture for 7 days in the presence of HCA (10-1,000 nM). Thus, HCA was found to possess anticancer effects in various types of human pancreatic cancer cells in vitro. This was a novel finding.

The suppressive effects of HCA on the proliferation of MIA PaCa-2 cells were determined in the presence of various inhibitors that induce cell cycle arrest in vitro (Fig. 3). The cells were cultured for 3 days in the absence (Fig. 3A) or presence (Fig. 3B) of HCA (100 nM) with or without butyrate $(10$ and $100 \mu \mathrm{M})$, roscovitine $(10$ and $100 \mathrm{nM})$ or sulforaphane (1 and $10 \mathrm{nM})(16,19,20)$. Proliferation of the MIA PaCa-2 cells was suppressed in the presence of these inhibitors (Fig. 3A). The suppressive effects of these inhibitors on cell proliferation were not observed in the presence of HCA (100 nM) (Fig. 3B).

To determine the involved mechanism by which HCA possesses suppressive effects on cell proliferation, we ascertained whether HCA regulates intracellular signaling pathways using various inhibitors for cell signaling. The suppressive effects of HCA on the proliferation of MIA PaCa-2 cells were not altered in the presence of TNF- $\alpha$, an enhancer of NF- $\kappa \mathrm{B}$ signaling (21), or Bay $\mathrm{K} 8644$, an agonist of $\mathrm{Ca}^{2+}$ entry in 

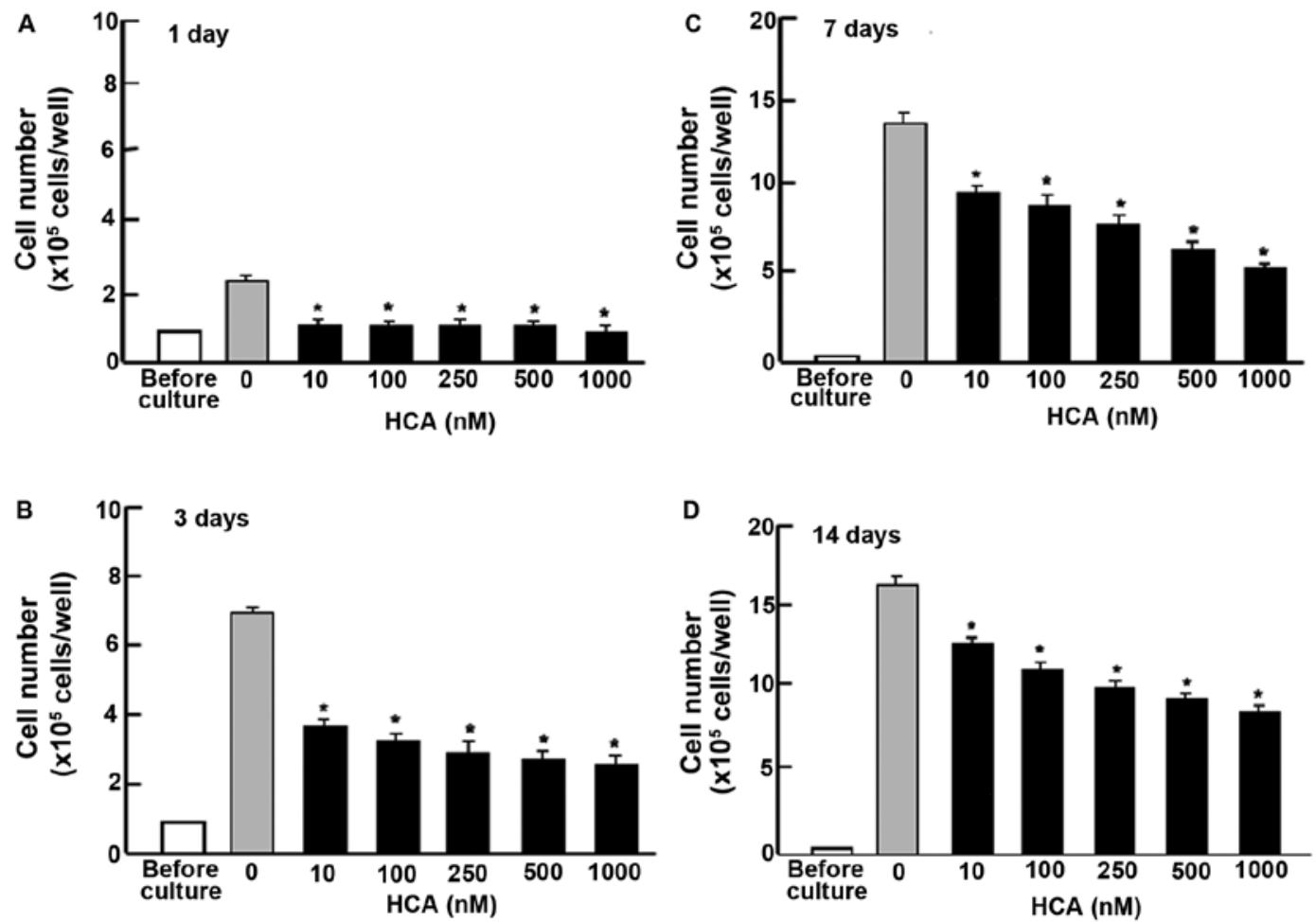

Figure 1. $p$-hydroxycinnamic acid (HCA) suppresses the proliferation of human pancreatic cancer MIA PaCa-2 cells in vitro. Cells were cultured in the presence or absence of HCA (10-1,000 nM) for 1 (A), 3 (B), 7 (C) or 14 (D) days. After culture, the number of attached cells on the dish was counted. Data are presented as mean $\pm \mathrm{SD}$ of 2 replicate wells per data set using different dishes and cell preparation. " $\mathrm{P}<0.001$ vs. control (grey bar); one-way ANOVA, Tukey-Kramer post test.
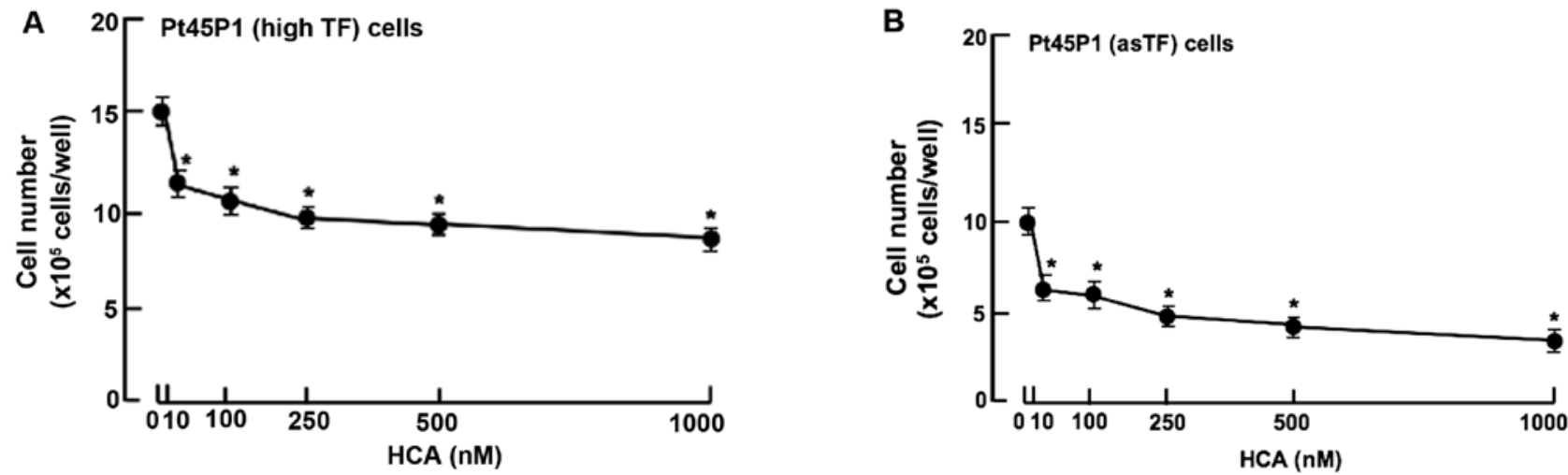

Figure 2. $p$-hydroxycinnamic acid (HCA) suppresses the proliferation in human pancreatic cancer Pt45P1 cells in vitro. Cells [A: highly expressing TF (high $\mathrm{TF}$ ) or B: highly expressing alternative spliced variant of TF (asTF)]were culture for 7 days in the absence or presence of HCA (10-1,000 nM). After culture, the number of attached cells on the dish was counted. Data are presented as mean \pm SD of 2 replicate wells per data set using different dishes and cell preparation. ${ }^{*} \mathrm{P}<0.001$ vs. control; one-way ANOVA, Tukey-Kramer post test.

cells (22) (Fig. 4A). The suppressive effects of HCA (100 nM) on the proliferation of MIA PaCa-2 cells were not modulated in the presence of PD98059 $(1 \mu \mathrm{M})$, an extracellular signal-regulated kinase (ERK) inhibitor (23) or staurosporin $(0.1 \mu \mathrm{M})$, an inhibitor of protein kinase C (24) (Fig. 4B). In addition, the suppressive effects of regucalcin on cell proliferation were not potentiated in the presence of wortmannin $(1 \mu \mathrm{M})$, an inhibitor of phosphatidylinositol 3-kinase (PI3K) (25) or DRB (1 $\mu \mathrm{M})$, an inhibitor of transcriptional activity with RNA polymerase II inhibition (26) (Fig. 4C). Thus, HCA was suggested to inhibit various processes involved in intracellular signaling that are related to cell proliferation.
Moreover, we compared the suppressive effects of HCA on human pancreatic cancer cells using gemcitabine, a potent antitumor agent that induces nuclear DNA damage (27). Culture with gemcitabine (50-1,000 nM) for 7 days (Fig. 5A) or 14 days (Fig. 5B) suppressed the proliferation of the MIA PaCa-2 (Fig. 5A) and Pt45P1 (high TF) (Fig. 5B) cells. Notably, the suppressive effects of HCA $(10 \mathrm{nM})$ on the proliferation of MIA PaCa-2 cells were significantly potentiated in the presence of gemcitabine $(10 \mathrm{nM})$ with a concentration that did not possess suppressive effects on cell proliferation (Fig. 5C). Such effects were not observed in the case of Pt45P1 (high TF) cells (Fig. 5D). 

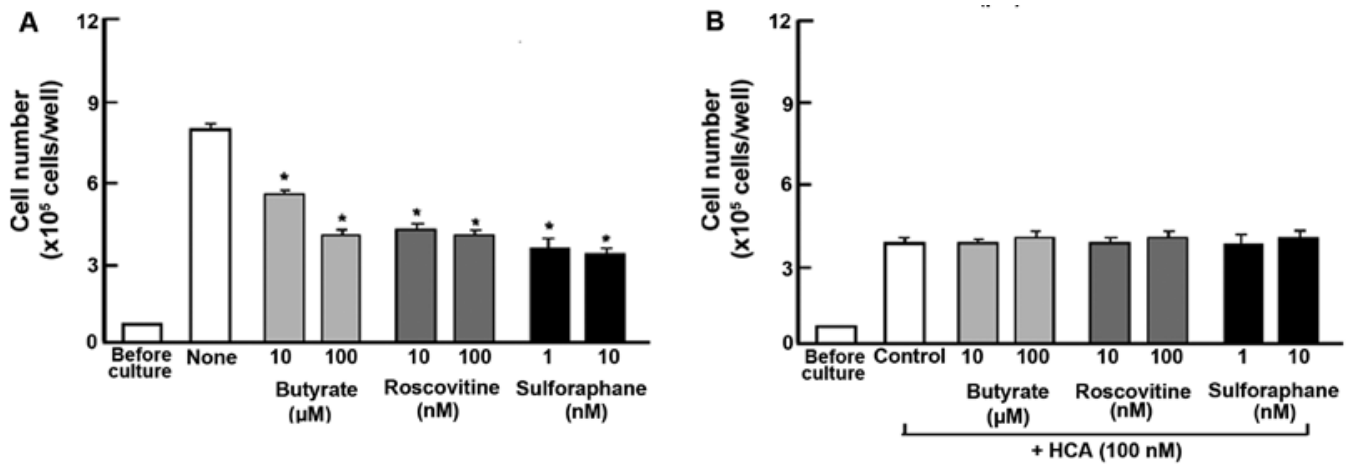

Figure 3. $p$-hydroxycinnamic acid (HCA) induces cell cycle arrest in the proliferation of human pancreatic cancer MIA PaCa-2 cells in vitro. Cells were culture for 3 days in the absence (A) or presence (B) of HCA (100 nM) with or without butyrate (10 and $100 \mu \mathrm{M})$, roscovitine (10 and $100 \mathrm{nM})$ or sulforaphane (1 and $10 \mathrm{nM})$. After culture, the number of attached cells on the dish was counted. Data are presented as mean \pm SD of 2 replicate wells per data set using different dishes and cell preparation. ${ }^{*} \mathrm{P}<0.001$ vs. control (white bar); one-way ANOVA, Tukey-Kramer post test.
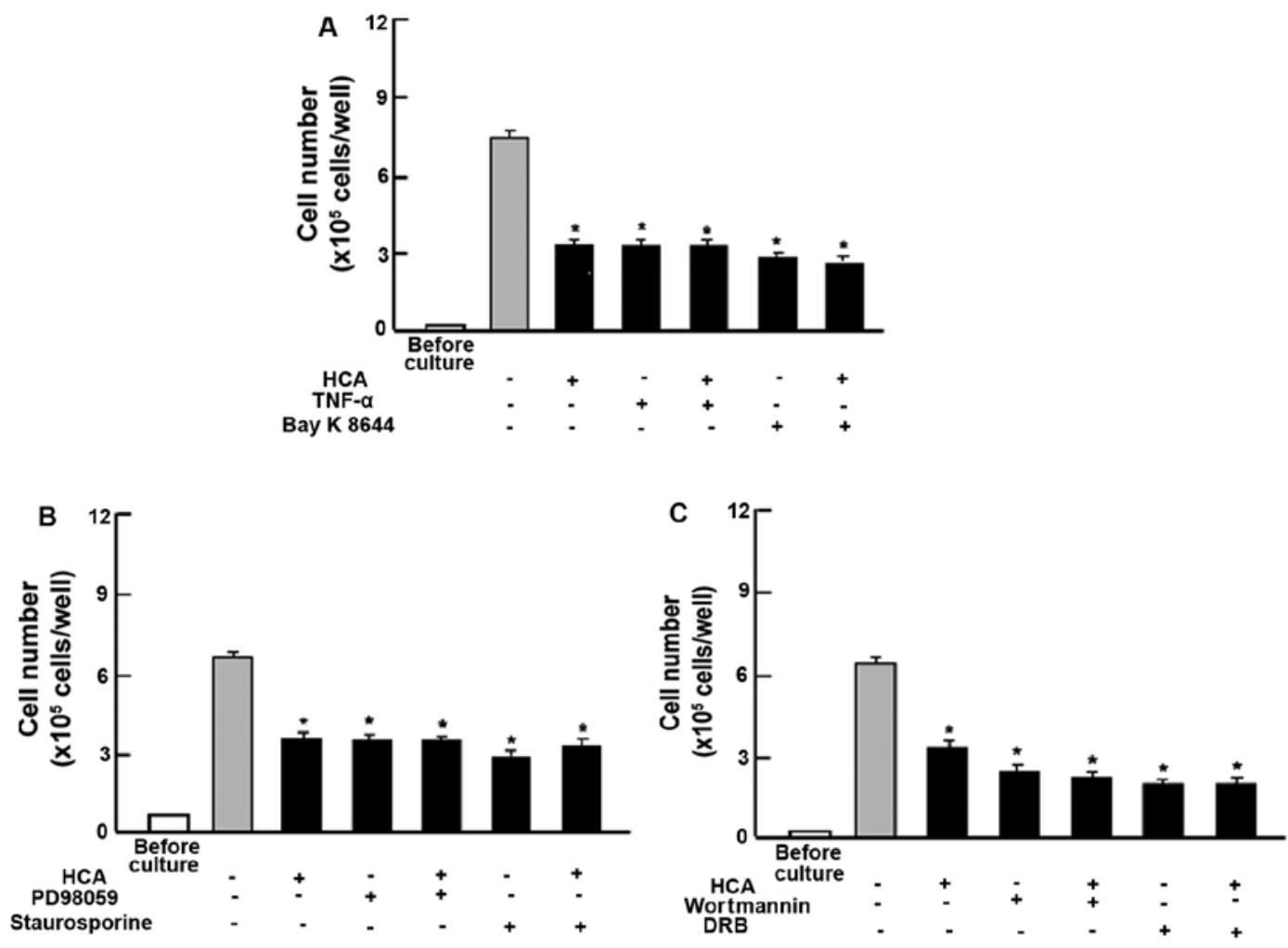

Figure 4. Suppressive effect of $p$-hydroxycinnamic acid (HCA) on proliferation is mediated through the inhibition of various signaling pathways in human pancreatic cancer MIA PaCa-2 cells in vitro. Cells were culture for 3 days in the presence of HCA (100 nM) with or without (A) TNF- $\alpha$ (1 ng/ml) or Bay $\mathrm{K} 8644(1 \mu \mathrm{M})$, (B) with or without PD98059 $(1 \mu \mathrm{M})$ or staurosporine $(0.1 \mu \mathrm{M})$, or $(\mathrm{C})$ with or without wortmannin $(1 \mu \mathrm{M})$ or DRB $(1 \mu \mathrm{M})$. After culture, the number of attached cells on the dish was counted. Data are presented as mean \pm SD of 2 replicate wells per data set using different dishes and cell preparation. ${ }^{*} \mathrm{P}<0.001$ vs. control (grey bar). One-way ANOVA, Tukey-Kramer post test.

HCA stimulates cell death. To determine the effects of HCA on cell death in human pancreatic cancer MIA PaCa-2 cells, the cells were cultured for 7 days. After reaching confluency, the cells were cultured for an additional 3 days in the presence of HCA (10-1,000 nM) (Fig. 6). Cell number was decreased after culture with HCA (10-1,000 nM) (Fig. 6A). Similar effects of HCA (10-1,000 $\mathrm{nM})$ on decreasing the cell number were also noted in human pancreatic cancer Pt45P1 (highly expressing TF) cells (Fig. 6B). The effects of HCA (10 or $100 \mathrm{nM})$ in decreasing the numbers of MIA PaCa-2 cells (Fig. 6C) and Pt45P1 (highly expressed TF) cells (Fig. 6D) were not potentiated in the presence of gemcitabine $(100 \mathrm{nM})$.
MIA PaCa-2 cells were cultured for 7 days. After reaching confluency, the cells were cultured for an additional 2 days in the presence of HCA (10 or $100 \mathrm{nM}$ ) with or without a caspase-3 inhibitor $(5 \mu \mathrm{M})$. The suppressive effects of HCA on cell death were prevented in the presence of the caspase-3 inhibitor (Fig. 7). Thus, HCA was suggested to stimulate cell death related to an increase in caspase-3 activity.

\section{Discussion}

The flavonoid HCA, which exhibits an anabolic effect on osteoporotic bone loss (6-8), has been demonstrated to stimulate 

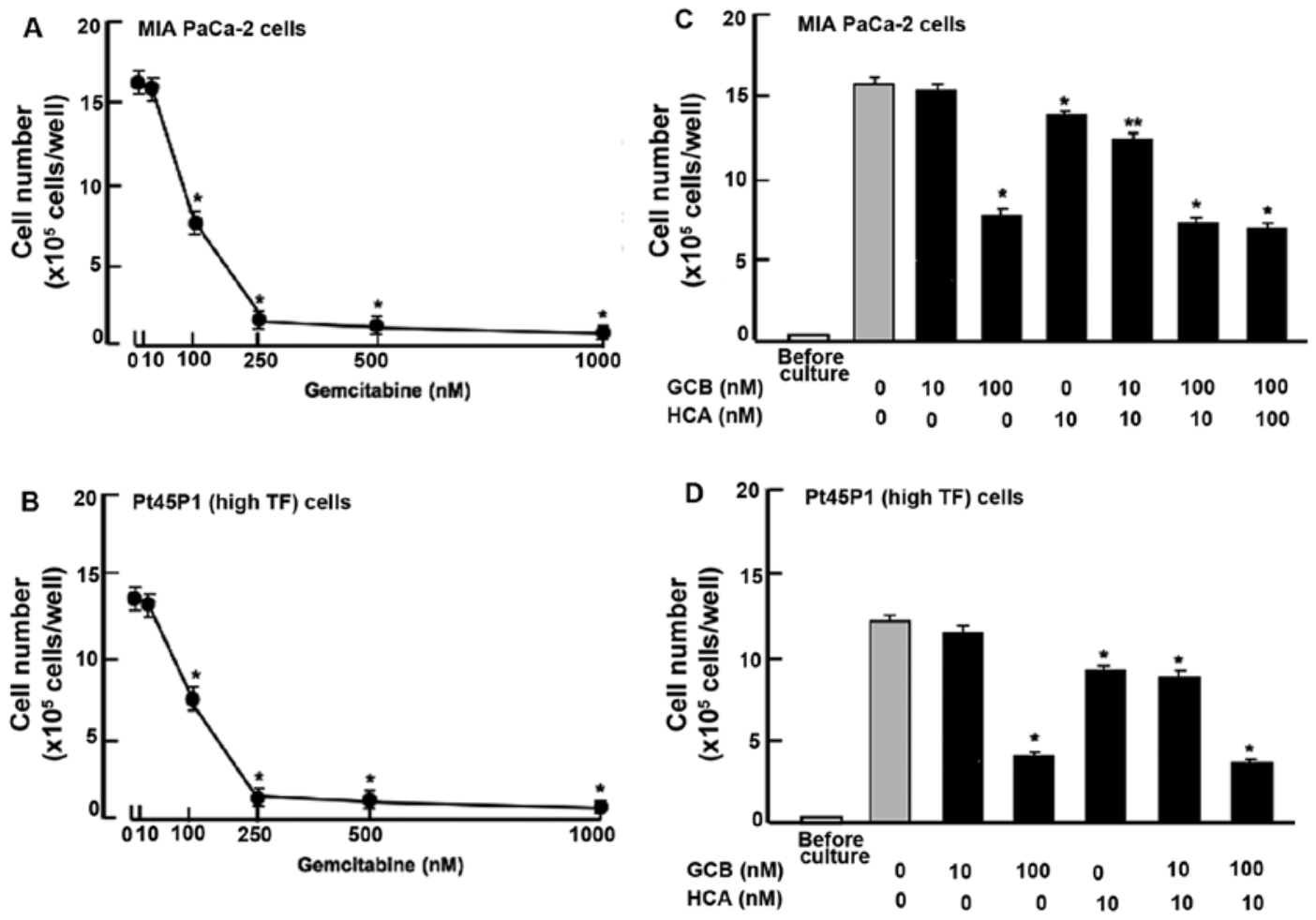

Figure 5. Comparison of the suppressive effects of $p$-hydroxycinnamic acid (HCA) and gemcitabine (GCB) on the proliferation of human pancreatic cancer MIA PaCa-2 or Pt45P1 (highly expressing TF) cells in the presence of GCB in vitro. (A) MIA PaCa-2 or (B) Pt45P1 (highly expressing TF) cells were cultured for 7 days in the presence of GCB (10-1,000 nM). (C) MIA PaCa-2 cells were cultured for 7 days in the presence of HCA (10 or $100 \mathrm{nM})$ with or without GCB $(10$ or $100 \mathrm{nM})$. (D) Pt45P1 (highly expressing TF) cells were cultured for 7 days in the presence of HCA (10 nM) with or without GCB (10 or $100 \mathrm{nM})$. After culture, the number of attached cells on the dish was counted. Data are presented as the mean \pm SD of 2 replicate wells per data set using different dishes and cell preparation. ${ }^{*} \mathrm{P}<0.001$ vs. control (grey bar). ${ }^{* *} \mathrm{P}<0.001$ vs. GCB $(100 \mathrm{nM})$ alone; one-way ANOVA, Tukey-Kramer post test.
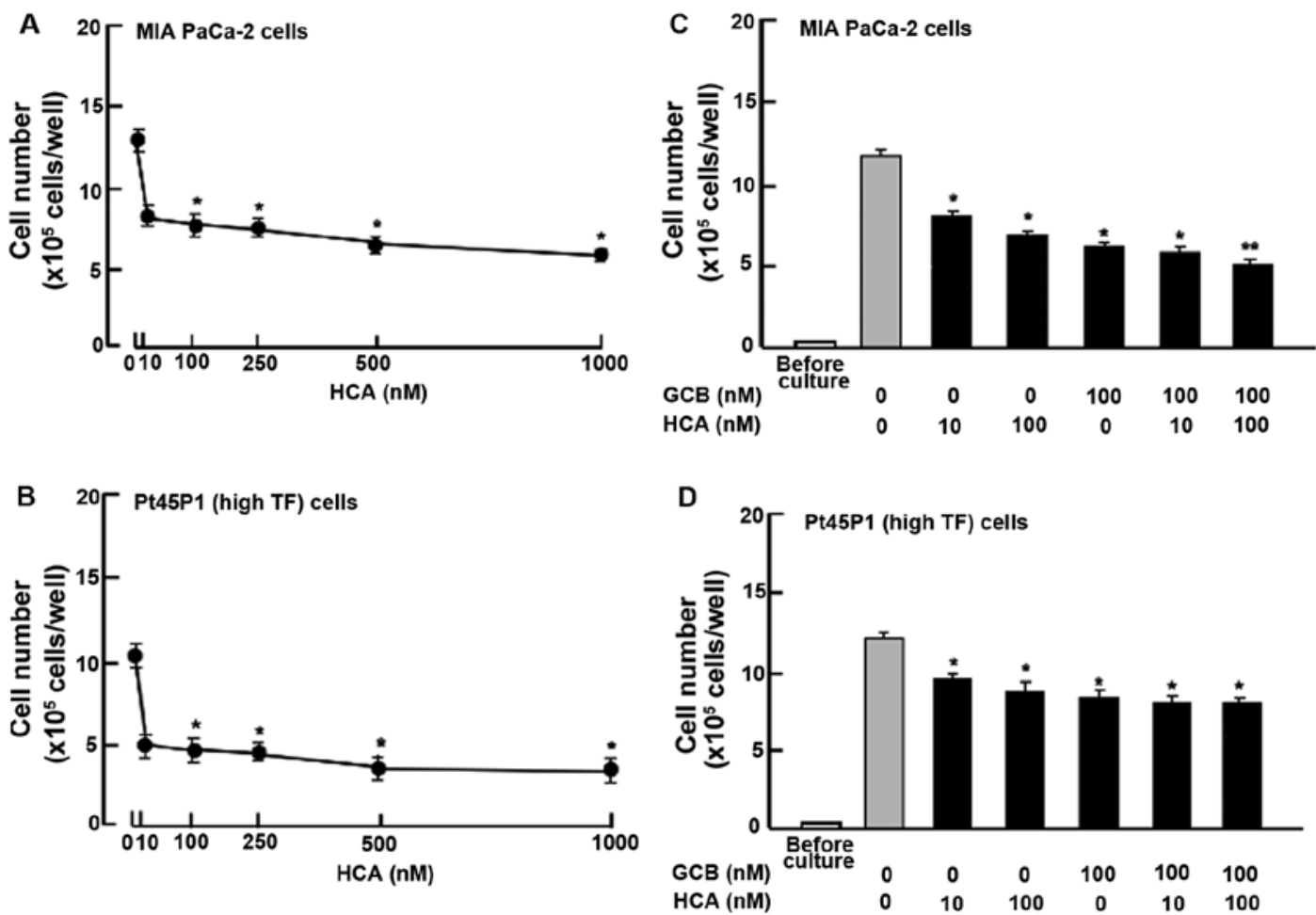

Figure 6. $p$-hydroxycinnamic acid (HCA) stimulates cell death in human pancreatic cancer MIA PaCa-2 and Pt45P1 (highly expressingTF) cells in vitro. (A) MIA PaCa-2 or (B) Pt45P1 cells were cultured for 7 days until reaching conflueny, and then the cells were cultured for an additional 3 days in the presence of HCA (10-1,000 nM). (C) MIA PaCa-2 cells or (D) Pt45P1 cells were cultured for 7 days until reaching confluency, and then the cells were cultured for an additional 3 days in the presence of HCA $(10$ or $100 \mathrm{nM})$ with or without gemcitabine (GCB) $(100 \mathrm{nM})$. After culture, the number of attached cells on the dish was counted. Data are presented as the mean $\pm \mathrm{SD}$ of 2 replicate wells per data set using different dishes and cell preparation. ${ }^{*} \mathrm{P}<0.001 \mathrm{vs.}$ the control (grey bar); one-way ANOVA, Tukey-Kramer post test. 


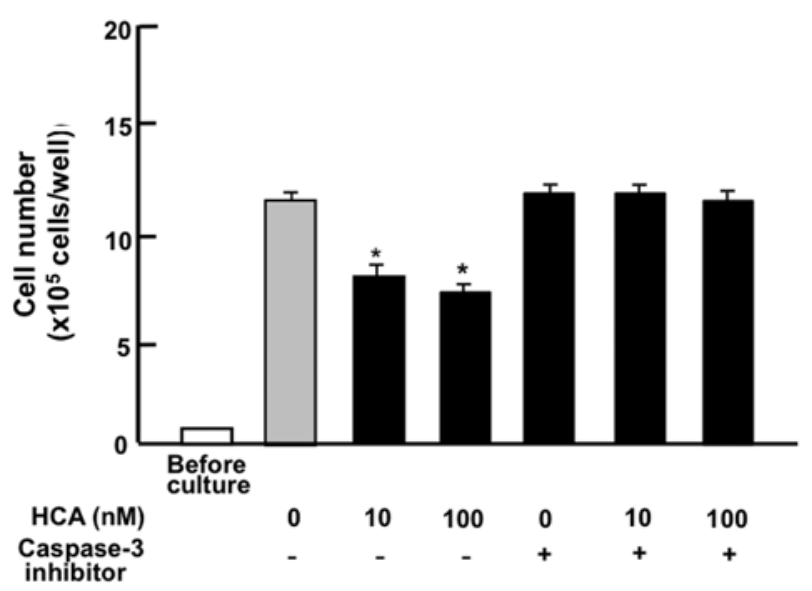

Figure 7. The stimulatory effects of $p$-hydroxycinnamic acid (HCA) on cell death are prevented in the presence of a caspase- 3 inhibitor in human pancreatic cancer MIA PaCa-2 cells in vitro. Cells were cultured for 7 days until reaching confluency, and then the cells were cultured for an additional 6 days in the presence of HCA (10 or $100 \mathrm{nM})$ with or without a caspase-3 inhibitor $(5 \mu \mathrm{M})$. After culture, the number of attached cells on the dish was counted. Data are presented as the mean \pm SD of 2 replicate wells per data set using different dishes and cell preparation. ${ }^{*} \mathrm{P}<0.001$ vs. control (grey bar); one-way ANOVA, Tukey-Kramer post test.

osteoblastic mineralization and to suppress osteoclastogenesis and adipogenesis in mouse bone marrow culture in vitro (9-12). In these processes, HCA has been shown to suppress various signaling pathways including $\mathrm{NF}-\kappa \mathrm{B}$ and MAPK/ERK that play a pivotal role in cell regulation of various types of cells. These signaling systems are known to be disturbed in cancer cells. We hypothesized that HCA may exhibit anticancer cell effects by restoring attenuated cell signaling in cancer cells.

HCA was demonstrated to suppress the proliferation and stimulate cell death in human pancreatic cancer MIA PaCa-2 and Pt45P1 (highly expressing TF) cells in vitro, supporting the view that HCA has an anticancer cell effect on various types of human pancreatic cancer cells. The suppressive effects of HCA on the proliferation of MIA PaCa- 2 cells were not potentiated in the presence of butyrate, roscovitine or sulforaphane that induce cell cycle arrest. Roscovitine is a potent and selective inhibitor of the cyclin-dependent kinase cdc $2, \mathrm{cdk} 2 \mathrm{~m}$ and cdk5 (19). Sulforaphane induces G2/M phase cell cycle arrest by stimulation of p21 and inhibition of cdc2 kinase (20). Butyrate induces inhibition of $\mathrm{G} 1$ progression by inhibiting Akt (16). HCA may induce G1 and G2/M phase cell cycle arrest by inhibiting the kinase activities of cdc2 and Akt in MIA PaCa-2 cells.

Moreover, the suppressive effects of HCA on MIA PaCa-2 cells were not modulated in the presence of various inhibitors that regulate intracellular signaling pathways in vitro. The suppressive effects of HCA on the proliferation of MIA PaCa-2 cells were not modulated in the presence of TNF- $\alpha$, an enhancer of $\mathrm{NF}-\kappa \mathrm{B}$ signaling (21), Bay K 8644, an agonist of $\mathrm{Ca}^{2+}$ entry in cells (22), PD98059, an inhibitor of ERK/mitogen-activated protein (MAP) kinase signaling pathway (23), staurosporin, an inhibitor of calcium-dependent protein kinase $\mathrm{C}$ signaling pathway (24) and wortmannin, an inhibitor of PI3/Akt signaling pathway (25). These findings suggest that HCA exerts suppressive effects mediated by the inhibition of various signaling pathways related to $\mathrm{NF}-\kappa \mathrm{B}, \mathrm{ERK}$, protein kinase $\mathrm{C}$, calcium signaling, or PI3K in MIA PaCa-2 cells. Moreover, the suppressive effects of HCA on cell proliferation were not enhanced in the presence of DRB, an inhibitor of transcriptional activity with RNA polymerase II inhibition (26). HCA may suppress transcriptional activity in the nucleus of MIA PaCa-2 cells. Thus, HCA may possess suppressive effects on the proliferation by inhibiting various signaling processes in human pancreatic cancer cells. HCA may be a multi-inhibitor in the proliferation of pancreatic cancer cells. Further research is warranted to elucidate the molecular mechanisms.

Gemcitabine is used clinically in the therapy of pancreatic cancer (27). Gemcitabine is a potent antitumor agent that induces nuclear DNA damage and apoptotic cell death in cancer cells (27). This agent suppresses cell proliferation and stimulates apoptotic cell death in cancer cells of various types (27). The stimulatory effects of HCA on cell death in the MIA PaCa-2 cells were significantly enhanced in the presence of gemcitabine. HCA may possess a different mode of action when compared to that of gemcitabine. HCA was also found to stimulate cell death in human pancreatic cancer Pt45P1 (highly expressing TF) cells in vitro. $\mathrm{HCA}$ at a comparative lower concentration (10 $\mathrm{nM})$ was found to exhibit suppressive effects on the proliferation and stimulatory effects on apoptotic cell death in the MIA PaCa-2 and Pt45P1 cells. Such effects were not observed at the same concentrations of gemcitabine. Notably, the stimulatory effects of HCA on cell death in the MIA PaCa- 2 cells were not noted in the presence of a caspase-3 inhibitor. HCA may stimulate cell death through a mechanism by which caspase-3 activity is increased. It is possible that HCA directly activates this enzyme in the nucleus. HCA, which is a botanical factor, may have lower toxicity as compared with that of gemcitabine. HCA may be a useful tool in the prevention and therapy in human pancreatic cancer.

In conclusion, this study demonstrates that the flavonoid HCA suppresses the proliferation and stimulates the cell death of human pancreatic cancer MIA PaCa-2 and Pt45P1 (highly expressing TF and alternative spliced variant TF) cells in vitro. Thus, HCA was found to have an anticancer effect in various types of human pancreatic cancer cells in vitro. This was a novel finding. HCA may be a useful tool in the prevention and therapy of human pancreatic cancers in vivo. Further research is needed to confirm the suppressive effects of HCA on carcinogenesis in vivo.

\section{References}

1. Sousa CM and Kimmelman AC: The complex landscape of pancreatic cancer metabolism. Carcinogenesis 35: 1441-1450, 2014.

2. Hidalgo M: Pancreatic cancer. N Engl J Med 362: 1605-1617, 2010.

3. McCarroll JA, Naim S, Sharbeen G, Russia N, Lee J, Kavallaris M, Goldstein D and Phillips PA: Role of pancreatic stellate cells in chemoresistance in pancreatic cancer. Front Physiol 5: 141, 2014.

4. Suzuki T, Nakayama H and Yamaguchi M: Effect of wasabi leafstalk (Wasabia japonica Matsum) extract on bone metabolism in mouse calvaria tissue culture. Food Sci Technol Int Tokyo 3: 366-369, 1997.

5. Suzuki T and Yamaguchi M: Purification of active component in wasabi leafstalk (Wasabia japonica Matsum) extract in stimulating bone calcification in vitro. J Health Sci 50: 483-490, 2004.

6. Lai YL and Yamaguchi M: Phytocomponent $p$-hydroxycinnamic acid stimulates bone formation and inhibits bone resorption in rat femoral tissues in vitro. Mol Cell Biochem 292: 45-52, 2006. 
7. Yamaguchi M, Lai YL, Uchiyama S and Nakagawa T: Oral administration of phytocomponent $p$-hydroxycinnamic acid prevents bone loss in ovariectomized rats. Mol Cell Biochem 311: 31-36, 2008.

8. Yamaguchi M, Uchiyama S and Lai YL: Oral administration of phytocomponent $p$-hydroxycinnamic acid has a preventive effect on bone loss in streptozotocin-induced diabetic rats. Int J Mol Med 19: 803-807, 2007.

9. Lai YL and Yamaguchi M: Phytocomponent $p$-hydroxycinnamic acid inhibits osteoclast-like cell formation in mouse bone marrow cultures. Int J Mol Med 19: 123-128, 2007.

10. Yamaguchi M, Lai YL, Uchiyama $S$ and Nakagawa $T$ : Phytocomponent $p$-hydroxycinnamic acid stimulates mineralization in osteoblastic MC3T3-E1 cells. Int J Mol Med 22: 287-291, 2008

11. Yamaguchi M and Weitzmann MN: The bone anabolic carotenoid $p$-hydroxycinnamic acid promotes osteoblast mineralization and suppresses osteoclast differentiation by antagonizing $\mathrm{NF}-\kappa \mathrm{B}$ activation. Int J Mol Med 30: 708-712, 2012.

12. Yamaguchi M, Baile CA, Zhu S and Shoji M: Bioactive flavonoid $p$-hydroxycinnamic acid stimulates osteoblastogenesis and suppresses adipogenesis in bone marrow culture. Cell Tissue Res 354: 743-750, 2013.

13. Ninomiya I, Yamazaki K, Oyama K, Hayashi H, Tajima H, Kitagawa H, Fushida S, Fujimura T and Ohta T: Pioglitazone inhibits the proliferation and metastasis of human pancreatic cancer cells. Oncol Lett 8: 2709-2714, 2014.

14. Sipos B, Möser S, Kalthoff H, Török V, Löhr M and Klöppel G: A comprehensive characterization of pancreatic ductal carcinoma cell lines: Towards the establishment of an in vitro reseach platform. Virchows Arch 442: 444-452, 2003.

15. Misawa $\mathrm{H}$, Inagaki $\mathrm{S}$ and Yamaguchi M: Suppression of cell proliferation and deoxyribonucleic acid synthesis in the cloned rat hepatoma H4-II-E cells overexpressing regucalcin. J Cell Biochem 84: 143-149, 2001.

16. Yamaguchi $M$ and Daimon Y: Overexpression of regucalcin suppresses cell proliferation in cloned rat hepatoma H4-II-E cells: Involvement of intracellular signaling factors and cell cycle-related genes. J Cell Biochem 95: 1169-1177, 2005.

17. Yamaguchi M: The anti-apoptotic effect of regucalcin is mediated through multisignaling pathways. Apoptosis 18: 1145-1153, 2013.
18. Yamaguchi M, Zhu S, Weitzmann MN, Snyder JP and Shoji M: Curcumin analog UBS109 prevents bone marrow osteoblastogenesis and osteoclastogenesis disordered by coculture with breast cancer MDA-MB-231 bone metastatic cells in vitro. Mol Cell Biochem 401: 1-10, 2015.

19. Meijer L, Borgne A, Mulner O, Chong JP, Blow JJ, Inagaki N, Inagaki M, Delcros JG and Moulinoux JP: Biochemical and cellular effects of roscovitine, a potent and selective inhibitor of the cyclin-dependent kinases cdc2, cdk2 and cdk5. Eur J Biochem 243: 527-536, 1997.

20. Singh SV, Herman-Antosiewicz A, Singh AV, Lew KL, Srivastava SK, Kamath R, Brown KD, Zhang L and Baskaran R: Sulforaphane-induced $\mathrm{G} 2 / \mathrm{M}$ phase cell cycle arrest involves checkpoint kinase 2-mediated phosphorylation of cell division cycle 25C. J Biol Chem 279: 25813-25822, 2004.

21. Li Y, Li A, Strait K, Zhang H, Nanes MS and Weitzmann MN Endogenous TNFalpha lowers maximum peak bone mass and inhibits osteoblastic Smad activation through NF-kappaB. J Bone Miner Res 22: 646-655, 2007.

22. Cano-Abad MF, Villarroya M, García AG, Gabilan NH and López MG: Calcium entry through L-type calcium channels causes mitochondrial disruption and chromaffin cell death. J Biol Chem 276: 39695-39704, 2001

23. Chen S, Wang Y, Ruan W, Wang X and Pan C: Reversing multidrug resistance in hepatocellular carcinoma cells by inhibiting extracellular signal-regulated kinase/mitogen-activated protein kinase signaling pathway activity. Oncol Lett 8: 2333-2339, 2014.

24. Chen QW, Edvinsson L and Xu CB: Role of ERK/MAPK in endothelin receptor signaling in human aortic smooth muscle cells. BMC Cell Biol 10: 52, 2009.

25. Serrano-Nascimento C, da Silva Teixeira S, Nicola JP, Nachbar RT, Masini-Repiso AM and Nunes MT: The acute inhibitory effect of iodide excess on sodium/iodide symporter expression and activity involves the PI3K/Akt signaling pathway. Endocrinology 155: 1145-1156, 2014.

26. Palangat M, Grass JA, Langelier MF, Coulombe B and Landick R: The RPB2 flap loop of human RNA polymerase II is dispensable for transcription initiation and elongation. Mol Cell Biol 31: 3312-3325, 2011.

27. Tang SC and Chen YC: Novel therapeutic targets for pancreatic cancer. World J Gastroenterol 20: 10825-10844, 2014. 